\title{
Synovial Neuronal Changes in Knee Joint Osteoarthritis
}

\author{
Haq Nawaz1, Masood Umer'1, Shahryar Noordin', Bo Christer Bertilson², Jian Li' \\ Aisha Siddiqah Ahmed ${ }^{4}$, Mahmood Ahmed ${ }^{2}$ \\ ${ }^{1}$ Department of Surgery, Aga Khan University, Karachi, Pakistan \\ ${ }^{2}$ Karolinska Institute, Department of Neurobiology, Care Sciences and Society, Huddinge, Sweden \\ ${ }^{3}$ Karolinska Institute, Department of Molecular Medicine and Surgery, Karolinska University Hospital, \\ Stockholm, Sweden \\ ${ }^{4}$ Karolinska Institute, Department of Clinical Neurosciences, Stockholm, Sweden \\ Email: aisha.ahmed@ki.se
}

Received 7 March 2016; accepted 24 May 2016; published 27 May 2016

Copyright (C 2016 by authors and Scientific Research Publishing Inc.

This work is licensed under the Creative Commons Attribution International License (CC BY).

http://creativecommons.org/licenses/by/4.0/

(c) (i) Open Access

\begin{abstract}
Purpose: The purpose is to study whether pain and inflammation in knee joint osteoarthritis (OA) are associated with local synovial neuronal changes. Methods: Synovial biopsies were harvested from the medial and lateral knee compartments from $\mathrm{OA}$ patients undergoing total joint replacement surgery. All patients had predominant pain at the medial joint compartment. Pain and knee joint function were evaluated by knee society score (KSS). Synovial inflammation was analyzed by histopathological analysis and expression of growth associated protein-43 (GAP-43), sensory (SP, CGRP) and autonomic (NPY, VIP, TH) neuropeptides was studied by single and double immunohistochemistry techniques. Results: We observed reduced KSS and increased inflammatory score in synovial membrane of medial knee compartment. A significant increase in GAP-43 [P=0.001], SP [P $=0.05]$, CGRP $[P=0.05]$ and $T H[P=0.05]$ expression was observed and SP, CGRP and NPY were found to be co-existed predominantly with GAP-43 in synovial membrane collected from medial compared to the lateral knee compartment. Conclusions: Regenerating nerve fibers containing sensory and autonomic neuropeptides are associated with pain and inflammation in knee joint $0 A$.
\end{abstract}

\section{Keywords}

Osteoarthritis, Knee Joint, Pain, Inflammation, Neuropeptides

\section{Introduction}

Osteoarthritis (OA) is the most prevalent painful joint disease leading to functional disability. Pain is the initial 
symptom followed by various degrees of changes in the cartilage and bone [1]. Patients with OA experience pain both in motion and at rest. Most widely used drugs, nonsteroidal anti-inflammatory drugs (NSAIDs), provide incomplete or temporary pain relief and in many cases are not effective [2] probably due to presence of multiple pain sources.

Role of synovial inflammation in development of joint pain and disease progression is implicated mainly by the release of various pro-inflammatory mediators [3] [4]. Free nerve endings are identified in the rat and human synovial membrane [5] [6] and probably play an important role in joint pain mediation. Increased expression of neuropeptides such as substance P (SP) and calcitonin gene-related peptide (CGRP) has been reported with increased synovial inflammation [6]. Increased SP expression was observed in the subchondral bone in knee and hip joints with OA [7] [8]. Moreover, up regulation of SP and CGRP was demonstrated in hip joint capsule and soft tissues in patients with painful OA [9]. Peripheral release of SP from nerve terminals has sensitizing effect on surrounding nociceptive afferents and stimulates immune cells to release pro-inflammatory cytokines. Interestingly, SP has been suggested to induce synthesis of inflammatory cytokines in neuroglial and lymphoid cells causing increase in pain sensitivity [10]. Further, a link between autonomic neuropeptides NPY, VIP and TH with the degree of synovial inflammation in rheumatoid arthritis (RA) and OA has been reported [11] [12]. Sprouting of sensory and autonomic nerve fibers has been suggested to occur in painful arthritic joint in experimental arthritis [13].

The present study was designed to examine the occurrence and distribution of growth associated protein 43 (GAP-43), a marker of regenerating nerve fibers in relation to pain and inflammation in knee OA, further, to observe sensory and autonomic neuropeptides expression in regenerated nerve fibres in synovial biopsies obtained from knee joint OA patients.

\section{Material and Methods}

\subsection{Patients}

A total of 13 patients with knee joint OA, 5 female and 8 male, mean age 68 years (57 - 78 years) undergoing total knee joint replacement surgery were included in the study. All patients were selected according to ACR criteria and were recruited during a period of one year at orthopedic outpatient clinic, Agha Khan University Hospital, Karachi, Pakistan. Patients were selected after more than 3 months anti-inflammatory treatments and physiotherapy without significant effect on knee joint pain. All patients had severe spontaneous pain and tenderness on the medial side of the knee joint with reduced walking ability. In all patients, OA was confirmed by weight bearing X-ray. Study was approved by local ethical research committee and informed consent was obtained from all individual participants included in the study. Patients with chronic pain syndromes and inflammatory rheumatic diseases were excluded from study.

\subsection{Pain Measurement}

Preoperatively, all patients were evaluated for pain, range of motion, stability and function of knee joint by using a knee society score (KSS) of the Hospital for Special Surgery [14]. The KSS reflects an objective measurement such as patient-reported pain severity (50 points), stability (25 points) and range of motion (25 points). Thus, 100 points will be obtained by a well-aligned knee with no pain, 125 of motion, and negligible antero-posterior and medio-lateral instability. Patient function considers walking distance (50 points) and stair climbing (50 points) with deductions for walking aids. The maximum function score 100 is obtained by a patient who can walk an unlimited distance and go up and down stairs normally [14].

\subsection{Histopathological Assessment}

Synovial samples $\left(6-8 \mathrm{~mm}^{2}\right.$ ) were obtained from medial and lateral compartments of OA knee during surgery. Tissues were immediately fixed in Zamboni's solution consisting of 4\% paraformaldehyde in 0.2 mol/l Sörensen phosphate buffer, pH 7.3, containing $0.2 \%$ picric acid. Sections were cut using a Leitz® 1720 cryostat (Ernst Leitz, Germany) to 7 or $14 \mu \mathrm{m}$ thickness and mounted on SuperFrost/Plus slides.

Two sections of each tissue at different depths (approximately $100 \mu \mathrm{m}$ apart) were taken and stained with haematoxylin and eosin for histological assessment. For semi-quantitative analysis, from each section 6 microscopic fields were taken. Thus, in all specimens a total of 12 microscopic fields were analyzed. Histologic find- 
ings were evaluated using a 10 point scoring system as described previously [6]. Briefly, synovial hyperplasia was graded on a scale of 0 to 1 , vascular changes on a scale of 0 to 2, villous hyperplasia on a scale of 0 to 3 and cellular infiltration was scored on 0 to 4 scales (Table 1 ).

\subsection{Immunohistochemical Assessment}

For SP, CGRP, NPY, VIP, TH and GAP-43 expression, $14 \mu \mathrm{m}$ sections were incubated overnight with antiserum to SP, (1:10000), CGRP (1:10000), NPY (1:5000) or VIP (1:5000, BACHEM Peninsula Laboratories; UK), TH (1:2000) and GAP-43 (1:2000, Chemicon International Inc., California). Sections were rinsed in PBS and incubated with biotinylated secondary goat anti-rabbit or horse anti-mouse antibodies both 1:250 (Vector Laboratories, Burlingame, California). Finally, Cy3-(1:5000) or Cy2-conjugated avidin (1:2000, Amersham Life Science Inc., USA) was used for visualization of immunoreactions. To demonstrate specificity of staining, primary antiserum were either omitted or pre-incubated with specific blocking peptides.

For double staining, after completing the staining step for GAP-43 using fluorochrome Cy2-conjugated avidin, sections were incubated with avidin followed by biotin-blocking solution. Sections were then incubated with antibody to SP, CGRP, NPY, VIP and TH before using biotinylated secondary horse anti-mouse antibodies (1:250, Vector Laboratories, California). Finally Cy3-conjugated avidin (1:2000, Amersham Life Science Inc., USA) was used for visualization of immunoreaction.

For semi-quantitative analysis, 2 sections from lateral and medial synovial compartment were stained and images of 6 microscopic fields from each tissue section were captured by a video camera system (DEI-750, Optronics) attached to microscope (Eclipse E800, Yokohama, Japan) and stored in computer. The immunoreactivity was measured as area (in $\mathrm{mm}^{2}$ ), using Easy Analysis software (Bergström Instruments, Sweden), as previously described [15]. A standard lower and upper threshold of fluorescence intensity was consistently applied for positively stained nerve fibers. The results were expressed as nerve fiber area (in $\mathrm{mm}^{2}$ ) in relation to the total area of each microscopic field.

\subsection{Statistical Analysis}

The Mann-Whitney U test was applied to analyse difference in histopathological score. Unpaired Student t-test was applied to verify between groups changes for immunohistochemistry analysis. Significance was set at P $\leq$ 0.05 .

Table 1. Ten-point scoring system to evaluate synovitis of osteoarthritic knee.

\begin{tabular}{|c|c|}
\hline \multicolumn{2}{|l|}{ Synovial lining cell hyperplasia } \\
\hline 1 - 2 layers of cells & 0 \\
\hline More than 3 layers & 1 \\
\hline \multicolumn{2}{|l|}{ Vascular changes } \\
\hline None & 0 \\
\hline Edema and vascular congestion & 1 \\
\hline Vascular proliferation & 2 \\
\hline \multicolumn{2}{|l|}{ Villous hyperplasia } \\
\hline Smooth surface & 0 \\
\hline Irregular & 1 \\
\hline Partially fingerlike hyperplasia & 2 \\
\hline Diffuse fingerlike hyperplasia & 3 \\
\hline \multicolumn{2}{|l|}{ Cellular infiltration } \\
\hline None & 0 \\
\hline Scattered cells & 1 \\
\hline Moderate; cell cluster & 2 \\
\hline Perivascular cell aggregation & 3 \\
\hline Marked cellular infiltration with lymphoid follicle & 4 \\
\hline Total score & 10 \\
\hline
\end{tabular}




\section{Results}

\subsection{Clinical Evaluation}

In all patients, KSS was considerably reduced with mean score observed as 15.4 for pain, 14.6 for motion and 15 points for knee stability. Knee function score was recorded as 22.3 for walking to 27.7 for stairs (Table 2).

\subsection{Histopathological Assessment}

Severe inflammatory changes were observed in the medial side of the knee compared to the lateral. Overall, a significant $[\mathrm{P}=0.004] 61 \%$ increase was observed in total histopathological score at the medial synovial side compared to the lateral side. A $45 \%$ increase in synovial hyperplasia score and in vascular changes and $35 \%$ increase in villous hyperplasia and cellular infiltration was observed in the medial synovial side compared to the lateral side (Figure 1(a), Figure 1(b); Table 3).

\subsection{Immunohistochemical Assessment}

Our immunohistochemical analysis revealed GAP-43, SP, CGRP, NPY, VIP and TH in the synovia obtained from all OA knee joint.

In the medial compartment a significant $[\mathrm{P}=0.001] 67 \%$ increase in GAP-43 expression was observed compared to lateral compartment. Nerve fibres immunoreactive to SP and CGRP were consistently found in

Table 2. Pre-operative clinical evaluation of OA patients by Knee Society Score (KSS). KSS scores based on clinical examination of knee joint and specific questionnaire regarding pain and functional capacity of knee. Data are presented as median with interquartile ranges with $\mathrm{n}=13$.

\begin{tabular}{ccc}
\hline Clinical assessment & Knee Society Score (KSS) & Maximum score \\
\hline Knee & & 50 \\
Pain & $15.4(10-30)$ & 25 \\
Range of motion & $14.6(10-25)$ & 25 \\
Stability & $15.0(10-20)$ & 50 \\
Function & & 50 \\
Walking & $22.3(20-25)$ & $27.7(20-30)$ \\
Stairs & & 50 \\
\hline
\end{tabular}
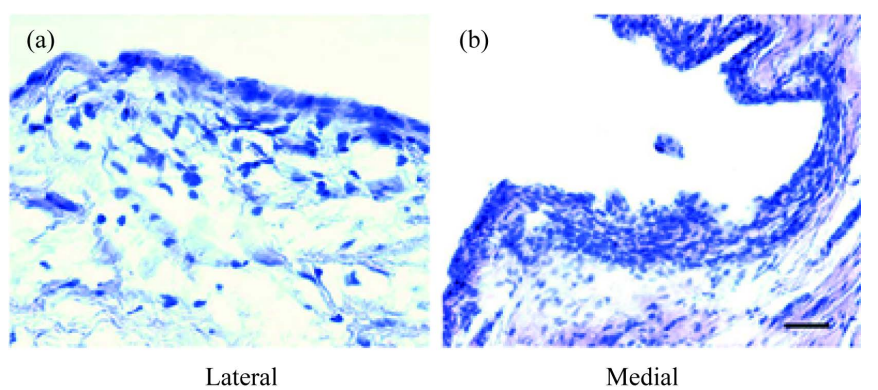

Figure 1. Photomicrographs of hematoxylin and eosin stained synovial membrane of the knee joint OA from lateral (a) and medial (b) compartments of the knee OA. Images are representative of $n=13$. (Original magnification $\times 20$; bar $=100 \mu \mathrm{m}$ )

Table 3. Histopathological evaluation of synovium from OA knee joint. Synovium was graded (0 - 10) according to pathological changes (Materials and Methods). Comparison of lateral and medial synovium: $* * \mathrm{P} \leq 0.005$. Data are presented as median with interquartile ranges. $\mathrm{n}=13$ in each group.

\begin{tabular}{lc} 
Groups & Synovitis score \\
\hline Lateral & $2(1.5-4)$ \\
Medial & $7(6-8)^{* *}$ \\
\hline
\end{tabular}


synovium from medial and lateral synovial side. SP-positive nerve fibres were arranged as thin mostly nonvascular, however, few SP-positive nerve fibres with varicosal endings were also observed. Semi-quantitative analysis demonstrated a significant $[\mathrm{P}=0.05] 63 \%$ increase in SP-immunoreactivity in medial compared to lateral side of the synovium. CGRP-positive nerve fibres were arranged both in nerve bundles and as single-nerves with varicosities mainly near blood vessels. Semi-quantitative analysis revealed $76 \%$ increase $[P=0.05]$ in the CGRP-immunoreactivity in medial side of the synovium (Figure 2(a), Figure 2(b)).

Among autonomic neuropeptides, nerve fibres immunoreactive to NPY, VIP and TH were consistently found in the synovium from both medial and lateral synovial compartments. However, semi-quantitative analysis showed a significant $[\mathrm{P}=0.05]$ increase in $\mathrm{TH}$-positive fibers by $51 \%$ in the medial compared to lateral synovial side (Figure 2(a), Figure 2(b)). No change in the NPY or VIP-positive nerve fibres was observed in the medial side compared to the lateral side of the synovium. NPY positive nerve fibres were found as networks around blood vessels.

Double staining analysis showed co-expression of SP and CGRP with GAP-43 in regenerating nerve fibers in both compartments but predominantly in medial synovial compartment. For NPY, we observed co-expression only in the medial side of synovia (Figures 3(a)-(e)). A weak co-expression was observed for VIP and TH with GAP-43 in all three compartments of synovia.

(a)

(a)
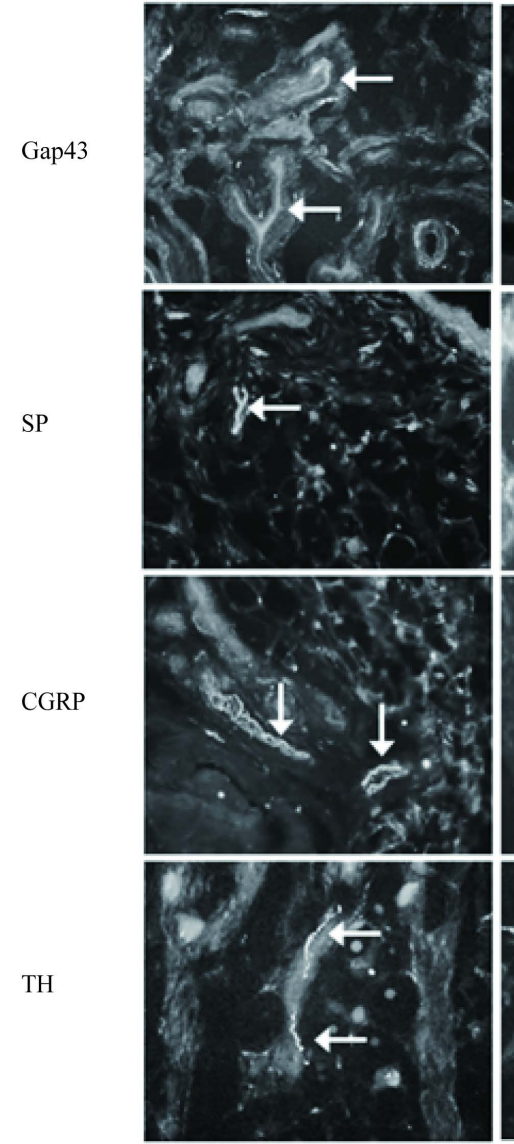
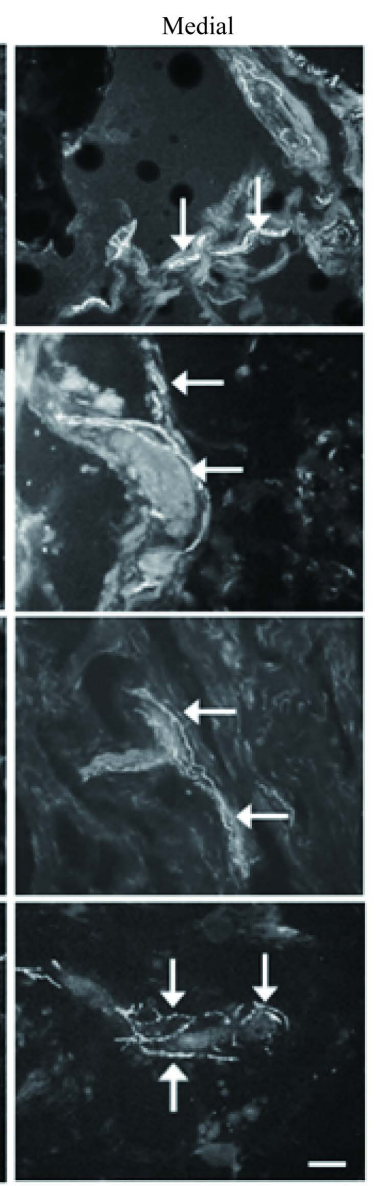

(b)
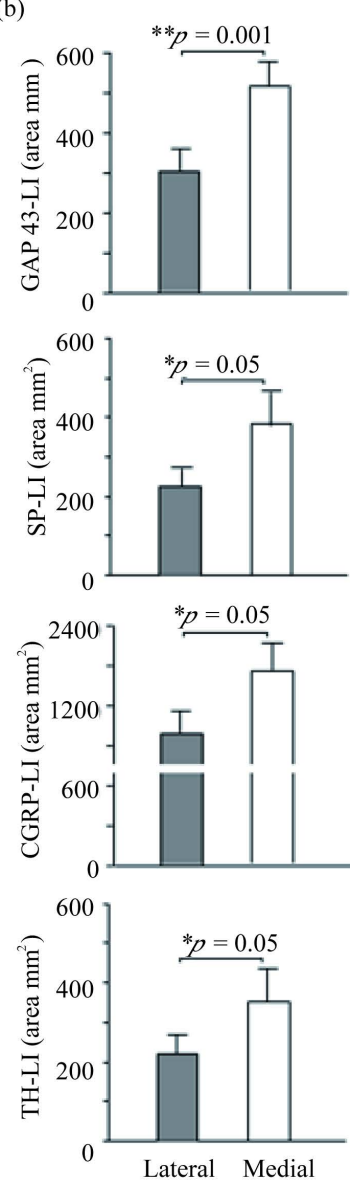

Figure 2. Immunofluorescence micrographs and semi-quantitative analysis of growth associated protein-43 (GAP-43), substance P (SP), calcitonin gene-related peptide (CGRP) and vasoactive intestinal protein (VIP) in OA knee synovia. (a) Nerve fibers positive for GAP-43, SP, CGRP and TH from the lateral and medial compartments from OA knee joint synovia (Original magnification $\times 20$; bar $=100 \mu \mathrm{m}$; arrows represent positive staining). (b) Semi-quantitative analysis of GAP-43, SP, CGRP and TH positive nerve fibers (immunofluorescent area $\mathrm{mm}^{2}$ ) in lateral and medial side from OA knee joint synovia. ${ }^{*} \mathrm{P} \leq 0.05 ;{ }^{* *} \mathrm{P} \leq 0.005$ comparison between the lateral and medial side of synovium by un-paired student $t$-test. Results are expressed as mean \pm SEM with $n=13$. 


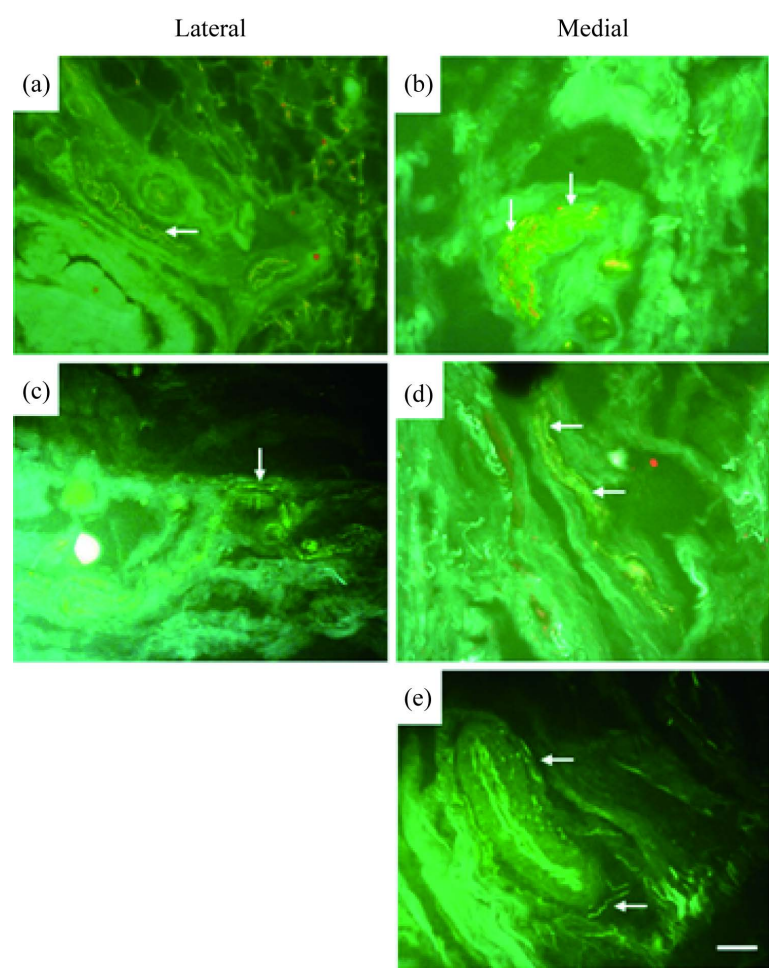

Figure 3. Fluorescence photomicrographs showing double staining of GAP-43 with SP (a, b) and CGRP (c, d) and NPY (e) from the lateral (a, c) medial (b, d, e) compartments from OA knee joint. Arrows show co-localization of GAP-43 with SP, CGRP and NPY (Original magnification $\times 20$; bar $=100 \mu \mathrm{m}$ ).

\section{Discussion}

Our present study indicates that pain and inflammation in patients with knee joint OA are related to neuronal changes in the synovia. Further, synovial inflammation along with sensory and autonomic nervous system exacerbates clinical symptoms in OA.

The previous concept of OA as disease explicitly of the cartilage is no longer valid as the degenerative processes involves the entire joint structure and most probably is accompanied by inflammatory signs leading to synovitis and joint pain [4] [16]. It is likely that overexpression of pro-inflammatory mediators in OA increases activation of pathophysiological pathways that contribute to progressive cartilage degradation. Patients included in the study experienced severe pain and tenderness and had reduced knee functions and knee stability as indicated by KSS. Correspondingly, our histopathological studies indicated severe inflammatory changes at the painful medial side of the knee joint compared to the lateral. Inflammatory changes such as synovial hyperplasia, vascular changes, cellular infiltration and villous hyperplasia were observed in the painful medial synovial side of OA knee.

Presence of synovial inflammation in patients with hip joint OA has been reported earlier along with the activation of several inflammatory cytokine [17]. Interestingly, the activation of one important transcription factor, nuclear factor kappa-beta (NF- $\kappa$ B) which is crucial for synthesis of inflammatory cytokines, has been observed in vascular endothelium and type A synovial lining cells of patients with RA and OA [18]. The pathological activation of NF- $\kappa$ B promotes transcription of genes which encode pro-inflammatory cytokines and matrix metalloproteases which ultimately lead to cartilage erosion. Interestingly, our recent pre-clinical observations of antagonizing NF- $\kappa \mathrm{B}$ resulted in reversal of pain behavior and knee joint inflammation which corresponded to the down regulation in MMP-3 and SP and CGRP expression in the rat model of OA [19].

In present study, we identified sensory (SP, CGRP) and autonomic (NPY, VIP, TH) nerves in synovial membrane retrieved from medial and lateral side of knee joint. We observed up-regulation of SP, CGRP and TH pos- 
itive nerve fibers in the medial painful side of knee compared to the lateral side. Similar observations are reported previously [6] however, our present results strengthen previous findings with semi-quantitative measurement of nerve fibers but also highlight changes in the autonomic system and explore the phenomenon of nerve regeneration in painful OA condition.

We identified nerve fibers positive to GAP-43 indicating nerve sprouting in inflamed synovial membrane especially in the painful medial side of knee joint. Presumably, growth of new nerves in inflamed tissue provides a delivery system for neuronal pro-inflammatory and nociceptive mediators, e.g., sensory and autonomic neuropeptides. Co-existence of GAP-43 with sensory and autonomic neuropeptides SP, CGRP and NPY further strengthens this notion. Interestingly, it has been reported that sensory and autonomic nerve fibers innervating aged knee joint maintain the capacity for nerve sprouting after inflammation or injury in mouse models [13] [20] [21]. The phenomenon of pathologic nerve regeneration in the human inflamed synovium as seen in the present study probably contributes to chronic joint pain and underlying changes in OA joints and may provide pharmacological insight and targets for better controlling pain in chronic musculoskeletal disorders.

\section{Study Limitations}

In present study, due to ethical issues we cannot include healthy tissues for neuropeptide expression as healthy synovial membrane cannot be collected. There is need of prospective studies where level of sensory and autonomic neuropeptides can be modified by physiotherapy and correlated to patients' pain. Number of patients should be increased for future studies.

\section{Acknowledgements}

Study was supported by grants from the Ulla och Gustaf af Ugglas Foundation, Sweden to ASA and grants from Aga Khan University to MA.

\section{Conflict of Interest}

Authors declare no professional or financial conflict of interest.

\section{References}

[1] Fernandes, J.C., Martel-Pelletier, J. and Pelletier, J.P. (2002) The Role of Cytokines in Osteoarthritis Pathophysiology. Biorheology, 39, 237-246.

[2] (2000) Recommendations for the Medical Management of Osteoarthritis of the Hip and Knee: 2000 Update. American College of Rheumatology Subcommittee on Osteoarthritis Guidelines. Arthritis \& Rheumatology, 43, 1905-1915.

[3] Smith, M.D., Triantafillou, S., Parker, A., Youssef, P.P. and Coleman, M. (1994) Synovial Membrane Inflammation and Cytokine Production in Patients with Early Osteoarthritis. The Journal of Rheumatology, 24, 365-371.

[4] Myers, S.L., Brandt, K.D., Ehlich, J.W., Braunstein, E.M., Shelbourne, K.D., Heck, D.A., et al. (1990) Synovial Inflammation in Patients with Early Osteoarthritis of the Knee. The Journal of Rheumatology, 17, 1662-1669.

[5] Buma, P., Elmans, L., Van Den Berg, W.B. and Schrama, L.H. (2000) Neurovascular Plasticity in the Knee Joint of An arthritic Mouse Model. The Anatomical Record, 260, 51-61. http://dx.doi.org/10.1002/1097-0185(20000901)260:1<51::AID-AR60>3.0.CO;2-9

[6] Saito, T. and Koshino, T. (2000) Distribution of Neuropeptides in Synovium of the Knee with Osteoarthritis. Clinical Orthopaedics and Related Research, 376, 172-182. http://dx.doi.org/10.1097/00003086-200007000-00024

[7] Mach, D.B., Rogers, S.D., Sabino, M.C., Luger, N.M., Schwei, M.J., Pomonis, J.D., et al. (2002) Origins of Skeletal Pain: Sensory and Sympathetic Innervation of the Mouse Femur. Neuroscience, 113, 155-166. http://dx.doi.org/10.1016/S0306-4522(02)00165-3

[8] Ogino, S., Sasho, T., Nakagawa, K., Suzuki, M., Yamaguchi, S., Higashi, M., et al. (2009) Detection of Pain-Related Molecules in the Subchondral Bone of Osteoarthritic Knees. Clinical Rheumatology, 28, 1395-402. http://dx.doi.org/10.1007/s10067-009-1258-0

[9] Saxler, G., Loer, F., Skumavc, M., Pfortner, J. and Hanesch, U. (2007) Localization of SP- and CGRP-Immunopositive Nerve Fibers in the Hip Joint of Patients with Painful Osteoarthritis and of Patients with Painless Failed Total Hip Arthroplasties. European Journal of Pain, 11, 67-74. http://dx.doi.org/10.1016/j.ejpain.2005.12.011

[10] Martin, F.C., Charles, A.C., Sanderson, M.J. and Merrill, J.E. (2009) Substance P Stimulates IL-1 Production by As- 
trocytes via Intracellular Calcium. Brain Research, 599, 13-18. http://dx.doi.org/10.1016/0006-8993(92)90846-2

[11] Miller, L.E., Grifka, J., Scholmerich, J. and Straub, R.H. (2002) Norepinephrine from Synovial Tyrosine Hydroxylase Positive Cells Is a Strong Indicator of Synovial Inflammation in Rheumatoid Arthritis. The Journal of Rheumatology, 29, 427-435.

[12] Hernanz, A., De Miguel, E., Romera, N., Perez-Ayala, C., Gijon, J. and Arnalich, F. (1993) Calcitonin Gene-Related Peptide II, Substance P and Vasoactive Intestinal Peptide in Plasma and Synovial Fluid from Patients with Inflammatory Joint Disease. Rheumatology, 32, 31-35. http://dx.doi.org/10.1093/rheumatology/32.1.31

[13] Ghilardi, J.R., Freeman, K.T., Jimenez-Andrade, J.M., Coughlin, K.A., Kaczmarska, M.J., Castaneda-Corral, G., et al. (2012) Neuroplasticity of Sensory and Sympathetic Nerve Fibers in a Mouse Model of a Painful Arthritic Joint. Arthritis \& Rheumatology, 64, 2223-2232. http://dx.doi.org/10.1002/art.34385

[14] Insall, J.N., Dorr, L.D., Scott, R.D. and Scott, W.N. (1989) Rationale of the Knee Society Clinical Rating System. Clinical Orthopaedics and Related Research, 248, 13-14. http://dx.doi.org/10.1097/00003086-198911000-00004

[15] Li, J., Kreicbergs, A., Bergstrom, J., Stark, A. and Ahmed, M. (2007) Site-Specific CGRP Innervation Coincides with Bone Formation during Fracture Healing and Modeling: A Study in Rat Angulated Tibia. Journal of Orthopaedic Research, 25, 1204-1212. http://dx.doi.org/10.1002/jor.20406

[16] Ayral, X., Pickering, E.H., Woodworth, T.G., Mackillop, N. and Dougados, M. (2005) Synovitis: A Potential Predictive Factor of Structural Progression of Medial Tibiofemoral Knee Osteoarthritis-Results of a 1 Year Longitudinal Arthroscopic Study in 422 Patients. Osteoarthritis and Cartilage, 13, 361-367. http://dx.doi.org/10.1016/j.joca.2005.01.005

[17] Takeshita, M., Nakamura, J., Ohtori, S., Inoue, G., Orita, S., Miyagi, M., et al. (2012) Sensory Innervation and Inflammatory Cytokines in Hypertrophic Synovia Associated with Pain Transmission in Osteoarthritis of the Hip: A Case-Control Study. Rheumatology (Oxford), 51, 1790-1795. http://dx.doi.org/10.1093/rheumatology/kes173

[18] Marok, R., Winyard, P.G., Coumbe, A., Kus, M.L., Gaffney, K., Blades, S., et al. (1996) Activation of the Transcription Factor Nuclear Factor-KappaB in Human Inflamed Synovial Tissue. Arthritis \& Rheumatology, 39, 583-591. http://dx.doi.org/10.1002/art.1780390407

[19] Ahmed, A.S., Li, J., Erlandsson-Harris, H., Stark, A., Bakalkin, G. and Ahmed, M. (2012) Suppression of Pain and Joint Destruction by Inhibition of the Proteasome System in Experimental Osteoarthritis. Pain, 153, 18-26. http://dx.doi.org/10.1016/j.pain.2011.08.001

[20] Jimenez-Andrade, J.M. and Mantyh, P.W. (2012) Sensory and Sympathetic Nerve Fibers Undergo Sprouting and Neuroma Formation in the Painful Arthritic Joint of Geriatric Mice. Arthritis Research \& Therapy, 14, R101. http://dx.doi.org/10.1186/ar3826

[21] Jimenez-Andrade, J.M., Mantyh, W.G., Bloom, A.P., Freeman, K.T., Ghilardi, J.R., Kuskowski, M.A., et al. (2012) The Effect of Aging on the Density of the Sensory Nerve Fiber Innervation of Bone and Acute Skeletal Pain. Neurobiology of Aging, 33, 921-932. 